\title{
THE ASYMPTOTIC DEVELOPMENTS OF A CLASS OF ENTIRE FUNCTIONS
}

\author{
H. K. HUGHES
}

1. Introduction. In an earlier paper [2], ${ }^{1}$ the author established a general theorem concerning the asymptotic behavior in the neighborhood of the point at infinity of an entire function $f(z)$ defined by a Maclaurin series of the form

$$
\sum_{n=0}^{\infty} g(n) z^{n}
$$

where the coefficient $g(n)$ of $z^{n}$ satisfies certain conditions. In the present paper, we wish to show an application of this theorem. We shall use it to find the asymptotic expansions of an important class of entire functions, namely those defined by the series

$$
\sum_{n=0}^{\infty} \frac{z^{n}}{\Gamma\left(\alpha_{1} n+s_{1}\right) \Gamma\left(\alpha_{2} n+s_{2}\right) \cdots \Gamma\left(\alpha_{m} n+s_{m}\right)} \quad(m \geqq 2),
$$

where $\alpha_{j}>0(j=1,2, \cdots, m)$ and $s_{j}$ are any constants, real or complex. Functions of this type are frequently connected with the solutions of linear differential equations. Moreover, a special case of (2) is the so-called generalized Bessel function, namely

$$
\phi(z)=\sum_{n=0}^{\infty} \frac{z^{n}}{n ! \Gamma(l n+p)}
$$

which has important applications in partition theory.

Throughout the paper, we shall denote by $f(z)$ the function defined by series (2), and by $g(n)$ the coefficient of $z^{n}$ in (2). Moreover, we shall adopt single symbols to represent certain combinations of the quantities $m, \alpha_{j}$, and $s_{i}$, these being as follows :

$$
\alpha=\sum_{j=1}^{m} \alpha_{j} ; \quad s=\sum_{j=1}^{m} s_{j} ; \quad \sigma=\alpha^{\alpha} \prod_{j=1}^{m}\left(\alpha_{j}\right)^{-\alpha(j)} ;
$$

$$
\gamma=\prod_{j=1}^{m}\left(\alpha_{j}\right)^{s(j)-1 / 2} ; \quad t=s+\frac{1}{2}-\frac{1}{2} m ; \quad c=\frac{\alpha^{t-3 / 2}}{(2 \pi)^{(m-1) / 2} \cdot \gamma} .
$$

Presented to the Society, November 25, 1944; received by the editors December 29, 1944.

${ }^{1}$ Numbers in square brackets apply to the references at the end of the paper.

${ }^{2}$ We use the symbol $\alpha(j)$ to denote $\alpha_{j}$ when the latter occurs as an exponent. The symbols $s(j)$ in (3) and $\beta(j)$, and so on, in (6) have a similar meaning. 
These six quantities enter prominently into the results to be established, and whenever any of the symbols $\alpha, s, \sigma, \gamma, t$, or $c$ appears in a formula, it will mean the corresponding quantity defined above.

2. Statement of results. It will be observed that the function $g(w)$, where $w=x+i y$, is single-valued and analytic in the finite $w$-plane. Furthermore, in $\S 4$, we shall show that, when considered throughout the arbitrary right half-plane $x>x_{0}, g(w)$ can be expressed in the form

$$
\begin{aligned}
g(w)=c \alpha \sigma^{w}\left\{\frac{1}{\Gamma(\alpha w+t)}+\frac{c_{1}}{\Gamma(\alpha w+t+1)}\right. & +\cdots \\
& \left.+\frac{c_{q}+\delta(\alpha w, q)}{\Gamma(\alpha w+t+s)}\right\},
\end{aligned}
$$

where $c_{1}, c_{2}, c_{3}, \cdots$ are determinate constants, and where $\lim _{|w| \rightarrow \infty} \delta(\alpha w, q)=0 ; q=0,1,2, \cdots$. Hence, according to the theorem proved in [2], the function $f(z)$, when considered for values of $z$ of large modulus such that $-\pi<\arg z \leqq \pi$, can be developed asymptotically in the form

$$
f(z) \sim c \sum_{\mu}\left\{Y_{\mu}^{(1-t)} \exp Y_{\mu} \sum_{n=0}^{\infty} c_{n}\left(Y_{\mu}\right)^{-n}\right\}-\sum_{n=1}^{\infty} g(-n) z^{-n},
$$

where $c_{0}=1, Y_{\mu}=(\sigma z)^{1 / \alpha} e^{2 \pi i \mu / \alpha}$, and $\sum_{\mu}$ denotes summation over those integral values of $\mu$ which satisfy the condition $|\arg z+2 \pi \mu| \leqq \pi \alpha / 2$.

If $0<\alpha<2$ and either $|\arg z|<\pi \alpha / 2$ or $|\arg z|>\pi \alpha / 2$, then (5) assumes a simpler form, as is shown in $[2, \S 4]$.

3. A special lemma. From what has been said in $\$ 2$, it is clear that the problem of determining the asymptotic expansion (5) of the function $f(z)$ is reduced to showing that the function $g(w)$ can be expressed in the form (4). For this purpose, we employ a certain lemma which will be introduced in this section.

Let $\Omega(w)$ represent the function

$$
\prod_{j=1}^{m}\left\{\frac{\Gamma\left(\beta_{j} w+b_{j}\right)}{\Gamma\left(\rho_{j} w+r_{j}\right)}\right\}
$$

where $\rho_{j}$ and $\beta_{j}$ are positive $(1 \leqq j \leqq m)$, while $b_{j}$ and $r_{j}$ are any constants, real or complex. Let $\rho, \beta, r$, and $b$ denote the respective sums $\sum_{j=1}^{m} \rho_{j}, \sum_{j=1}^{m} \beta_{j}, \sum_{j=1}^{m} r_{j}$, and $\sum_{j=1}^{m} b_{j}$, and let

$$
\theta=\prod_{j=1}^{m} \frac{\left(\beta_{j}\right)^{\beta(j)}}{\left(\rho_{j}\right)^{\rho(j)}} ; \quad \lambda=\prod_{j=1}^{m} \frac{\left(\beta_{j}\right)^{b(j)-1 / 2}}{\left(\rho_{j}\right)^{r(j)-1 / 2}} .
$$


Then the lemma with which we are concerned is as follows :

LEMMA. If $\rho=\beta$ and $r=b$, then for values of $w$ of large modulus such that $|\arg w|<\pi$, the function $\Omega(w)$ can be developed asymptotically in the form

$$
\Omega(w) \sim \lambda \theta^{w}\left\{1+\frac{c_{1}}{(w+1)}+\frac{c_{2}}{(w+1)(w+2)}+\cdots\right\}
$$

where $c_{1}, c_{2}, c_{3}, \cdots$ are determinate constants.

This lemma is a generalization of a simpler one employed by Ford $[1$, p. 74$]$ in his discussion of functions of Bessel type, and its proof follows the same line of reasoning. ${ }^{3}$ In fact, if we let

$$
\Psi(w)=\Omega(w) / \lambda \theta^{w},
$$

take the logarithm of both sides, and proceed as in the reference, we arrive at the relation

$$
\Psi(w) \sim 1+\frac{c_{1}}{(w+1)}+\frac{c_{2}}{(w+1)(w+2)}+\cdots,
$$

which is valid for large $|w|$ such that $|\arg w|<\pi$, and from which the lemma follows immediately. As for the values of the $c$ 's, they can be calculated from the formula

$$
c_{n}=\lim _{|w| \rightarrow \infty}(w+1)(w+2) \cdots(w+n) R_{n}(w),
$$

where

$$
R_{n}(w)=\Psi(w)-1-\sum_{q=1}^{n-1} \frac{c_{q}}{(w+1)(w+2) \cdots(w+q)} .
$$

4. Application to the function $g(w)$. In this section, we shall employ the lemma just established to prove relation (4). We shall denote by $\omega_{1}$ the product appearing in the denominator of $g(w)$; that is,

$$
\omega_{1}=\prod_{j=1}^{m} \Gamma\left(\alpha_{j} w+s_{j}\right)
$$

We begin by writing $\omega_{1}$ in the special form

$$
\omega_{1}=\omega_{1}\left(w^{\prime}\right)=\prod_{j=1}^{m} \Gamma\left(\frac{\alpha_{j} w^{\prime}+s_{j} \alpha+\alpha_{j}-\alpha_{j} t}{\alpha}\right),
$$

${ }^{8}$ Ford's lemma has been generalized in another manner by H. Van Engen. See Concerning gamma function expansions, Tohoku Math. J. vol. 45 (1939) pp. 124-129. 
where $\alpha$ and $t$ are as defined in (3), $w^{\prime}=\alpha w+t-1$, and where the notation $\omega_{1}\left(w^{\prime}\right)$ means that $w^{\prime}$, rather than $w$, is being regarded as the argument. Then we form the function

$$
W\left(w^{\prime}\right)=\Gamma\left(w^{\prime}+1\right) / \omega_{1}\left(w^{\prime}\right) .
$$

Applying Gauss' multiplication theorem for the gamma function to $\Gamma\left(w^{\prime}+1\right)$, and substituting in (10), we have

$$
\begin{aligned}
& W\left(w^{\prime}\right)=\frac{m^{w^{\prime}+1 / 2}}{(2 \pi)^{(m-1) / 2}} \frac{\omega_{2}\left(w^{\prime}\right)}{\omega_{1}\left(w^{\prime}\right)} ; \\
& \omega_{2}\left(w^{\prime}\right)=\prod_{j=1}^{m} \Gamma\left(\frac{w^{\prime}+j}{m}\right) .
\end{aligned}
$$

It is easily verified that the fraction $\omega_{2}\left(w^{\prime}\right) / \omega_{1}\left(w^{\prime}\right)$ when regarded as a function $\Omega\left(w^{\prime}\right)$ of $w^{\prime}$ satisfies the hypothesis of the lemma in $\S 3$. Hence it can be represented asymptotically when $\left|\arg w^{\prime}\right|<\pi$ in the form

$$
\Omega\left(w^{\prime}\right) \sim \lambda \theta^{w^{\prime}}\left[1+\frac{c_{1}}{\left(w^{\prime}+1\right)}+\frac{c_{2}}{\left(w^{\prime}+1\right)\left(w^{\prime}+2\right)}+\cdots\right],
$$

where the $c$ 's are determined as indicated in (8). In (12), $\lambda$ and $\theta$ have special values which are given by the products (6) in which $\rho_{j}, \beta_{j}, r_{j}, b_{j}$ are now equal to $\alpha_{j} / \alpha, 1 / m, s_{j}-\alpha_{j}(t-1) / \alpha$ and $j / m$ respectively. They are found to be

$$
\begin{gathered}
\lambda=\left(\frac{\alpha}{m}\right)^{1 / 2} \prod_{j=1}^{m}\left(\alpha_{j}\right)^{-p(j)} ; \quad p(i)=s_{j}-1 / 2-\alpha_{j}(t-1) / \alpha, \\
\theta=\frac{\alpha}{m} \prod_{j=1}^{m}\left(\alpha_{j}\right)^{-\alpha(j) / \alpha} .
\end{gathered}
$$

Having established (12), we next recall from (10) that

$$
g(w)=1 / \omega_{1}=\frac{W\left(w^{\prime}\right)}{\Gamma\left(w^{\prime}+1\right)} ; \quad w^{\prime}=\alpha w+t-1 .
$$

From (11), (12), and (13), it follows that $g(w)$ can be represented asymptotically in terms of $w^{\prime}$ in the form

$$
g(w) \sim C(m \theta)^{w^{\prime}} \sum_{n=0}^{\infty} \frac{c_{n}}{\Gamma\left(w^{\prime}+n+1\right)} ; \quad c_{0}=1,
$$

where $C$ denotes the constant $m^{1 / 2} \lambda /(2 \pi)^{(m-1) / 2}$. If we now replace $w^{\prime}$ 
by its value $w^{\prime}=\alpha w+t-1$, and make simple reductions, the last relation above assumes the form

$$
g(w) \sim c \alpha \sigma^{w}\left\{\frac{1}{\Gamma(\alpha w+t)}+\frac{c_{1}}{\Gamma(\alpha w+t+1)}+\cdots\right\} .
$$

This relation is valid when $|\arg w|<\pi$; hence relation (4) is seen to hold throughout any right half-plane $x>x_{0}$, and this fact is what we set out to prove.

As was remarked previously, the asymptotic expansion (5) of the function $f(z)$ defined by series (2) follows at once as soon as (4) has been established.

5. Applications to special functions. The general result stated in $\$ 2$ and established in the succeeding sections can be applied to find the asymptotic expansions of the function

$$
\phi(z)=\sum_{n=0}^{\infty} \frac{z^{n}}{n ! \Gamma(l n+p)}
$$

which was mentioned in $\S 1$. It will be noticed that the value of $g(-n)$ $(n=1,2,3, \cdots)$ is zero in this case. The values of the quantities $\alpha$ and $t$ are found by (3) to be $1+l$ and $p+1 / 2$ respectively, while $\sigma$ and $c$ are as follows :

$$
\sigma=\frac{(1+l)^{1+l}}{l^{l}}, \quad c=\frac{(1+l)^{p-1}}{(2 \pi)^{1 / 2} l^{p-1 / 2}} .
$$

The function $\phi(z)$ has been studied previously by E. M. Wright [4] whose results appear comparable with the present results if only the dominant terms in (5) are retained. They involve a sequence of constants $\left\{a_{n}\right\}$ analogous to the sequence $\left\{c_{n}\right\}$ in (5), but which are found by a considerably different process.

As a second application of the present results, we may consider an important class of integral functions recently studied by C. V. Newsom [3], namely

$$
F(z)=\sum_{n=0}^{\infty} g(n) z^{m n}
$$

where $m$ is a positive integer and $g(n)$ has the form

$$
g(n)=1 / \prod_{j=1}^{m} \Gamma\left(n+s_{j}\right)
$$

In applying the present result to $F(z)$, we must replace $z$ in (5) by $z^{m}$, and it is seen that the resulting expansion holds in the sector 
$-\pi / m<\arg z \leqq \pi / m$. In this case, we have $\alpha=m, t=\sum_{j=1}^{m} s_{j}+1 / 2-m / 2$, $\sigma=m^{m}$ and $c=m^{t-3 / 2}(2 \pi)^{1 / 2-m / 2}$. The result is substantially the same as that obtained by Newsom.

In conclusion, we may remark that certain extensions of the results established in the present paper are evidently possible. For example, the method can apparently be applied if a function $h(n)$, properly restricted, is put in the numerator of the coefficient of $z^{n}$ in (2).4 Moreover, an extension of both the method and the result would seem possible in case some of the numbers $\alpha_{j}$ in (2) were negative, sufficient of them remaining positive so that $f(z)$ is entire.

\section{REFERENCES}

1. W. B. Ford, The asymptotic developments of functions defined by Maclaurin series, University of Michigan Studies, Scientific Series, vol. 11, 1936.

2. H. K. Hughes, On the asymptotic expansions of entire functions defined by Maclaurin series, Bull. Amer. Math. Soc. vol. 50 (1944) pp. 425-430.

3. C. V. Newsom, The asymptotic behavior of a class of entire functions, Amer. J. Math. vol. 65 (1943) pp. 450-454.

4. E. M. Wright, The asymptotic expansion of the generalized Bessel function, Proc. London Math. Soc. vol. 38 (1935) pp. 257-270.

Purdue University

${ }^{4}$ See for example [1, Arts. 20 and 26]. 